\title{
Review: tardive dyskinesia is a risk factor for mortality in psychiatric patients
}

Ballesteros J, González-Pinto A, Bulbena A. Tardive dyskinesia associated with higher mortality in psychiatric patients: results of a meta-analysis of seven independent studies.J Clin Psychopharmacol 2000 Apr;20:188-94.

QUESTION: In psychiatric patients with tardive dyskinesia (TD), what is the risk of all cause mortality?

\section{Data sources}

Studies were identified by searching Medline (1966 to September 1998), Dissertation Abstracts (1992 to July 1998), ERIC (1982 to March 1998), PsycLIT (1993 to June 1998), and Current Contents (July 1995 to September 1998) using the terms dyskinesia, tardive dyskinesia, and mortality; and by scanning the references of retrieved articles.

\section{Study selection}

Studies were selected if they were prospective or retrospective, controlled or uncontrolled studies that specifically dealt with TD and mortality.

\section{Data extraction}

Data were extracted on study design, TD criteria, control for possible confounders, and length of follow up.

\section{Main results}

7 studies were included: 5 were prospective and 2 were retrospective; 2 studies were uncontrolled. Follow up ranged from 16 months to 15 years. No evidence of heterogeneity existed among the studies. The combined fixed effects odds ratio (OR) showed a statistically significant association between TD and mortality (OR $1.40,95 \%$ CI 1.16 to 1.69 , p $<0.001$ ). Prospective controlled (3 studies) and uncontrolled studies (2 studies) each showed an association (OR 1.4, CI 1.1 to $1.7, \mathrm{p}=0.002$ and OR 2.2 , CI 1.1 to 4.1 , $\mathrm{p}=0.02$, respectively) and retrospective controlled studies (2 studies) showed no association (OR 0.9, CI 0.3 to $2.3, \mathrm{p}=0.80)$. When the largest study $(\mathrm{n}=408)$ with the longest follow up (15 y) was removed in a sensitivity analysis, the overall effect estimate was not appreciably changed (OR 1.9, CI 1.2 to 3.0, $\mathrm{p}=0.006)$.

\section{Conclusion}

In psychiatric patients with tardive dyskinesia, the risk of all cause mortality is increased.

\section{COMMENTARY}

Increased mortality in people with mental illness may arise directly from the use of antipsychotic drugs and has led to a large scale study of the relation between psychotropic medication and sudden death. ${ }^{1}$ The meta-analysis by Ballesteros et al suggests that the development of TD is associated with a modest increase in mortality. Unfortunately, methodological weaknesses in the referent studies limit the clinical interpretation of the meta-analysis. TD is difficult to distinguish from age related involuntary movements and dyskinesia related to psychiatric illness. ${ }^{2}$ Nevertheless, the weak association shown in this study between all cause mortality and TD is both important and potentially worrying given the medicolegal aspects surrounding the prescription of neuroleptic drugs, and requires an explanation.

Severe TD could directly result in increased mortality through exhaustion, falls, poor nutrition, dysphagia, and pneumonia. Furthermore, TD has been associated with age, negative symptoms in schizophrenia, female sex, cumulative dose of neuroleptic, duration of exposure, brain injury, affective symptoms, and drug induced parkinsonism. ${ }^{3}$ A combination of these factors may explain the present finding of increased mortality associated with TD.

Further prospective cohort studies are needed to critically analyse the proposed association between mortality and TD, controlling for a wide range of variables and providing detailed information, ideally confirmed by post mortem, on cause of death. Whether the use of atypical neuroleptics will result in a reduction in the burden of TD remains to be determined. ${ }^{45}$

Jolyon Meara, MD

Peter Hobson, BSc University of Wales College of Medicine Glan Clwyd Hospital, Rhyl, UK

1 Appleby L, Shaw J, Amos T, et al. Sudden unexplained death in psychiatric in-patients. Br J Psychiatry 2000;176:405-6.

2 Morgenstern H, Glazer WM, Niedzwiecki D, et al. The impact of neuroleptic medication on tardive dyskinesia: a meta-analysis of published studies. Am J Public Health 1987;77:717-24.

3 Jeste DV. Tardive dyskinesia in older patients.J Clin Psychiatry 2000;61(suppl 4):27-32.

4 Beasley CM, Dellva MA, Tamura RN, et al. Randomised double-blind comparison of the incidence of tardive dyskinesia in patients with schizophrenia during long-term treatment with olanzapine or halperidol. Br J Psychiatry 1999;1 14:23-30.

5 Davidson M, Harvey PD, Vervarcke J, et al. A long-term, multicenter, open-label study of risperidone in elderly patients with psychosis. Int J Geriatr Psychiatry 2000;15:506-14.
Source of funding: no external funding.

For correspondence DrJ Ballesteros, University of the Basque Country, Medical School, Department of Neuroscience, Campus de Leioa s/n,E-48940 Leioa, Spain. Fax +34 944649266 (Work 1) $+34944700958$

(Work 2). 